\title{
Pulmonary Complications of Connective Tissue Disease.
}

\author{
Athol Wells \\ Christopher P. Denton \\ Royal Brompton Hospital and UCL Division of Medicine
}

Semin Respir Crit Care Med 40(2):145-146 Apr 2019

The current issue of Seminars in Respiratory and Critical Care Medicine reviews the spectrum of pulmonary complications in connective tissue diseases (CTDs), with a major focus on interstitial lung disease (ILD) and a detailed discussion of pulmonary vascular, airways and extrapulmonary disease processes. The first three articles cover histopathologic findings, imaging patterns and the integration of clinical, imaging and histopathologic findings in diagnosis, severity staging, monitoring and management. Pulmonary hypertension in CTDs is reviewed in a stand-alone piece. The remaining articles consist of in-depth reviews of pulmonary complications in selected individual CTDs.

Pulmonary complications, including ILD and pulmonary hypertension are the major source of mortality in most CTDs. The evaluation and management of patients with known or suspected CTD-ILD now makes up a significant proportion (up to $25 \%$ of cases) of the routine clinical practice of specialist ILD clinicians. Pulmonologists are often consulted when pulmonary complications are well-established. By contrast, many rheumatologists screen for the presence of ILD and pulmonary vascular disease in the hope that earlier intervention might be more effective. Whilst this 
approach undoubtedly benefits some patients, there is often difficulty in striking the optimal balance between immediate intervention and careful monitoring when minor abnormalities are disclosed. The articles in this issue provide a reference text of the range of abnormalities across the individual CTD-ILDs, allowing findings in individual patients to be seen in a broader perspective.

Importantly, the nature, prevalence and severity of pulmonary complications differs radically between individual CTDs, despite the tendency to group CTD-ILD cases in broader ILD cohorts. All of the entities within the current idiopathic interstitial pneumonia classification also occur in patients with CTD but there is considerable variation in their relative prevalence: usual interstitial pneumonia is the most frequent fibrotic histologic pattern in rheumatoid arthritis (RA) but non-specific interstitial pneumonia is more prevalent in other CTDs. The relentless progression seen in many patients with lung involvement in rheumatoid arthritis (RA) can be contrasted with the often self-limited ILD course in Sjogrens' syndrome (SS). Understanding the markers and drivers of ILD progression represents an important challenge that is likely to be unravelled best by looking across different autoimmune rheumatic diseases. Patients with ILD in inflammatory myopathy (IM) often have major pulmonary inflammation (organizing pneumonia) overlapping with diffuse alveolar damage, leading to extensive residual fibrosis in some cases, a profile seen infrequently in the other CTDs. The entity of undifferentiated CTD causes particular management difficulties with ongoing debate as to whether "interstitial pneumonitis with autoimmune features" is a valid diagnostic entity that should inform management.

Exactly the same variability across the CTDs is evident in the presence and severity 
of other pulmonary complications. Pulmonary hypertension, a malignant prognostic determinant, is a frequent consideration in some CTDs (especially systemic sclerosis [SSc] and systemic lupus erythematosus [SLE]), but less so in others. In RA and SLE, the range of pulmonary complications is much wider than in other CTDs. In most CTDs, there are often major disparities between the severity of pulmonary complications and the intensity of systemic disease activity. However, pulmonary manifestations in the anti-phospholipid syndrome pose unique challenges due to the need to identify and manage systemic involvement in parallel.

Awareness of the striking differences in pulmonary disease profiles between the CTDs is central to prognostic evaluation, based on the identification of individual disease processes and the staging of their severity by the integration of clinical, imaging, histologic, pulmonary function and echocardiographic findings. This, in turn, allows realistic therapeutic goals to be formulated, despite the paucity of controlled treatment data in this field. Recent treatment advances and the limitations of the evidence base are reviewed in individual CTDs, informing clinicians as to the rationale and consensus views on specific interventions.

In summary, we hope that this issue of Seminars will provide a road-map for clinicians in the identification, evaluation and management of the plethora of pulmonary disease processes that complicate the CTDs. The authors bring unrivalled collective experience of CTD management from respiratory medicine, rheumatology and cardiology that is testament to the central importance of multidisciplinary and cross-speciality working that is essential to good managment. We thank the contributors to this issue for their wisdom, experience and broad perspectives which, we believe, provide invaluable guidance in this extraordinarily 
difficult area. 